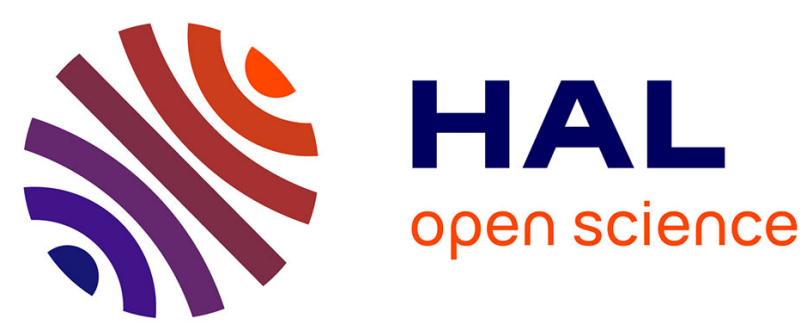

\title{
gmvBRAVE: A Powerful Tool for SBAS Engineering
}

Carlos Cuesta-Martínez, Eric Arnal, Javier Arenas, Begoña Ochoa

\section{To cite this version:}

Carlos Cuesta-Martínez, Eric Arnal, Javier Arenas, Begoña Ochoa. gmvBRAVE: A Powerful Tool for SBAS Engineering. ITSNT 2018, International Technical Symposium on Navigation and Timing, Oct 2018, Toulouse, France. 10.31701/itsnt2018.08 hal-01942242

\section{HAL Id: hal-01942242 \\ https://hal-enac.archives-ouvertes.fr/hal-01942242}

Submitted on 5 Dec 2018

HAL is a multi-disciplinary open access archive for the deposit and dissemination of scientific research documents, whether they are published or not. The documents may come from teaching and research institutions in France or abroad, or from public or private research centers.
L'archive ouverte pluridisciplinaire HAL, est destinée au dépôt et à la diffusion de documents scientifiques de niveau recherche, publiés ou non, émanant des établissements d'enseignement et de recherche français ou étrangers, des laboratoires publics ou privés. 


\title{
gmvBRAVE: \\ A powerful tool for SBAS engineering
}

\author{
C. Cuesta-Martínez, E. Arnal, J. Arenas, B. Ochoa, GMV \\ Email: cfcuesta@gmv.com
}

\section{BIOGRAPHIES}

Carlos Cuesta-Martínez is currently working on the development and maintenance of the gmvBRAVE tool. Besides, he is working as a software engineer in GMV within the EGNOS CPF-PS project. He received his $\mathrm{PhD}$ in Physics at the University of Valencia in 2017.

Eric Arnal holds a MSc in Aerospace Engineering by the University of California, Irvine (UCI). He joined GMV in 2014 and currently manages a SBAS-related project and is the coordinator of $g m v B R A V E$ activities.

Javier Arenas holds a MSc in Aerospace Engineering by the Universidad Politécnica de Madrid (UPM). He joined GMV in 2012. Currently, he works in different SBASrelated projects as an expert on performances and processing algorithms.

Ms Begoña Ochoa is the Head of the European SBAS Section, in GNSS Augmentation System and Services Division. She obtained her Masters in Mathematics by the Universidad Complutense de Madrid (UCM). She joined GMV in 1998 and she has participated from this time in different GMV activities regarding SBAS and other navigation systems like Galileo.

\footnotetext{
ABSTRACT

GMV presents gmvBRAVE (Brief Report for Analysis, Validation and Engineering platform), a powerful tool for SBAS engineering. SBAS systems are in great demand since they have revealed to be very useful not only for air navigation but for applications in many other fields. This fact has enabled that many countries have taken the leap to develop their own systems, and thus, the necessity of having an engineering tool that allows the complete monitoring of the performances and algorithms of a SBAS system, both for its development and operational stages, has become crucial. $g m v B R A V E$ is a tool that has been designed with such purpose: to contribute to improve the process of analyzing and reporting daily SBAS performances. gmvBRAVE automatizes the execution of SBAS algorithms and performance postprocessing tools to ease the generation of thorough and visual reports. Combined with a web interface to ease the visualization of the huge amount of results generated, $g m v B R A V E$ is a versatile tool that provides its users with
}

the required information for the comparison of different SBAS versions. This comparison functionality is one of the strengths of the gmvBRAVE and gives development teams a unique opportunity for increasing the productivity with the identification of anomalous events. Furthermore, it facilitates the development of evolutions and improvements of SBAS algorithm that can be tested continuously for long time intervals by using available real data.

The potential benefits that $g m v B R A V E$ can provide as a tool for engineering and performance analysis for the current and future development, deployment and maintenance of any SBAS system will be shown through this paper.

\section{INTRODUCTION}

Nowadays, combining Global Satellite Navigation Systems (GNSS) and augmentation systems is required in order to improve accuracy and assure integrity for safetyof-life applications. In particular, Satellite-Based Augmentation Systems (SBAS) are used in civil aviation and maritime. Over the recent years, the usage of SBAS has spread world-wide and its demand has risen in many other different sectors such as agriculture, geodesy or construction, among many others, where high-precision navigation services are also required.

The European SBAS, the so-called European Geostationary Navigation Overlay Service (EGNOS), together with WAAS in the USA, MSAS in Japan and GAGAN in India are the current four SBAS systems in operation around the world. The appearance of new GNSS constellations in addition to GPS and GLONASS, such as Galileo from Europe and BeiDou from China, and the introduction of the GPS civil L5 signal are imminent. In parallel, EGNOS system prepares for its next step forward, EGNOS V3 evolution, which will turn it into a Dual-Frequency Multi-Constellation (DFMC) system. Furthermore, the future EGNOS expansion to African regions (ASECNA), and the development of other SBAS systems (SDCM in Russia and BDSBAS in China), some of them already being deployed in test mode (e.g., TestBed in Australia and New Zealand), will mark a new milestone in the SBAS history. For all of that, having an easy but powerful tool that allows developers, maintainers and platform users to analyze and assess the performance 
of any SBAS system, from its design to its operational stages, has become a necessity.

Now, GMV has covered this need thanks to gmvBRAVE, the Brief Report for Analysis, Validation and Engineering platform [1].

The following paper presents the main features and different uses of $g m v B R A V E$ and it is organized as follows. In Section 2, gmvBRAVE is introduced in brief, where some of its functionalities (Section 2.1) and use cases (Section 2.2) are highlighted. In Section 3, its internal structure, data generation and data flow in the platform are presented. In Section 4 , the main features of gmvBRAVE's web interface are introduced. More in depth, Sections 4.1 and 4.2 show, respectively, some details about the daily analysis and historical performances sections of the web interface. In Section 5, practical cases of the usage of the gmvBRAVE tool are shown. In Section 5.1, the tool is used for SBAS maintenance activities. In Section 5.2, the tool is used to track and analyze the impact of anomalous events, while in Section 5.3 it is used as a tool for testing and validating experimental versions of SBAS algorithms which may improve the system behaviour against these anomalous events (that can be catalogued; see Section 5.4) and against other situations. Finally, Section 6 gives a brief conclusion about the benefits of using gmvBRAVE as an engineering tool for assessing the performances of any SBAS.

\section{GMVBR AVE IN A NUTSHELL}

$g m v B R A V E$ was initially conceived as a tool for obtaining relevant parameters from a SBAS system for a preliminary performance analysis, mostly in the cases of an event degrading SBAS expected performances. These parameters were obtained by automatizing different tools of analysis. Today, gmvBRAVE has become a powerful platform that enables the evaluation of the performances of any SBAS system, thus being the perfect tool to monitor any SBAS system during the different stages it may go through: design, requirements consolidation, validation and maintenance.

\subsection{Functionalities}

The gmvBRAVE's main functionalities and capabilities are:

- The automation of different SBAS engineering tasks such as core algorithm execution as well as algorithm qualification tests;

- The execution of scenarios using data from real receivers (e.g. archived or from the Internet), or generated synthetically (e.g. using GMV's EETES simulator [2]);

- The execution of long-time-period scenarios (covering several months or years) with barely any human effort, only the additional cost of hardware, storage and computing power resources;
- The management of different configurations for a single scenario;

- The monitoring of performances in terms of integrity, continuity, accuracy and availability, for several service levels (from Non-Precision Approach (NPA) to Approach with Vertical Guidance (APV) and SBAS CAT-I approaches), through a user-friendly web interface;

- The comparison at a glance of system performances (at pseudorange, user or algorithmic level) between different SBAS algorithm versions, including legacy (GPS L1) and DFMC, and for different configurations;

- The possibility of analyzing directly the SBAS messages from Signal in Space;

- The generation of daily reports with a configurable subset of information;

- The generation and storage of a historical archive of performances.

\subsection{Use cases}

Thanks to the previously mentioned functionalities, gmvBRAVE allows its users to perform many different activities among which are:

- Support to SBAS definition, development and maintenance phases;

- $\quad$ SBAS performance monitoring and analysis;

- Testing and validation of SBAS algorithm experimental versions in baseline scenarios;

- Testing safety barriers against anomalous events, showing in an easy manner the effect of each defined barrier;

- Cataloguing real data anomalous events.

\section{INTERNAL STRUCTURE \& DATA FLOW}

The internal structure of $g m v B R A V E$ is composed of welldifferentiated modules that allows development and maintenance teams to easily track any single issue that may arise during an execution and modify every single feature of it in a straightforward way, with the final goal of simplifying its maintenance.

To reach the functionalities and capabilities mentioned in the previous section, gmvBRAVE combines under a unique platform several GMV tools and services, which are:

- SBAS algorithms: core algorithms in charge of the computation of the corrections and integrity information characteristic of a SBAS, which can be of an operational, a test-bed (e.g. magicSBAS [3]) or any in-development SBAS system ready to be tested and validated.

- eclayr [4]: an analysis tool, fully developed and commercialized by GMV, which assesses the 
performance of a SBAS in terms of integrity, continuity, accuracy and availability. It processes SBAS information and reference GNSS and ionosphere data to generate performance assessment reports.

- Additional analysis utilities: customizable analysis tools which post-process internal data from SBAS algorithms and eclayr performance analyses.

- Web data utilities: interface to SBAS, GNSS and ionospheric archive systems through protocols FTP, NTRIP, etc., for instance via EDAS for EGNOS data [5].

Through its main launching command, gmvBRAVE offers the possibility to configure the whole execution by selecting:

- Tools to be executed;

- Scenario and data configuration;

- SBAS algorithm to use;

- Server to run the post-processing analysis;

- Webpage to which the results are uploaded;

- Mode of execution.

The internal workflow of the whole execution process of $g m v B R A V E$ is shown in Figure 1, where the different modules responsible of each task that involves the previously described tools can be differentiated.

First, the SBAS algorithm is executed with the provided configuration and automatically downloading the necessary input data. Second, once the SBAS algorithm module execution finishes, post-processing begins by executing, in parallel, both eclayr and the custom analysis tools. For the eclayr execution, the inputs are sent to an array of servers for its processing. Using ancillary data generated by eclayr and internal data from the SBAS algorithms, the custom analysis tools generate additional plots. From the moment the results begin to be generated, they are updated on the webpage.

The execution of gmvBRAVE generates lots of information that is finally displayed in three different formats:

a) Plots: graph figures are the main source of information provided to the platform user. They conveniently display data grouped from several sources to present illustrative figures.

b) Tables: some performance parameters are better shown in data tables. The storage of this numerical information allows tracking the evolution of these parameters as a function of time, having the possibility of also displaying this information through dynamic graphs.

c) Miscellaneous: other information unrelated to SBAS performance or internal algorithm behavior is displayed to the user in several formats that may include: usage of platform resources, status of queued and current executions, configuration and metadata of each execution, description of algorithms, and more.

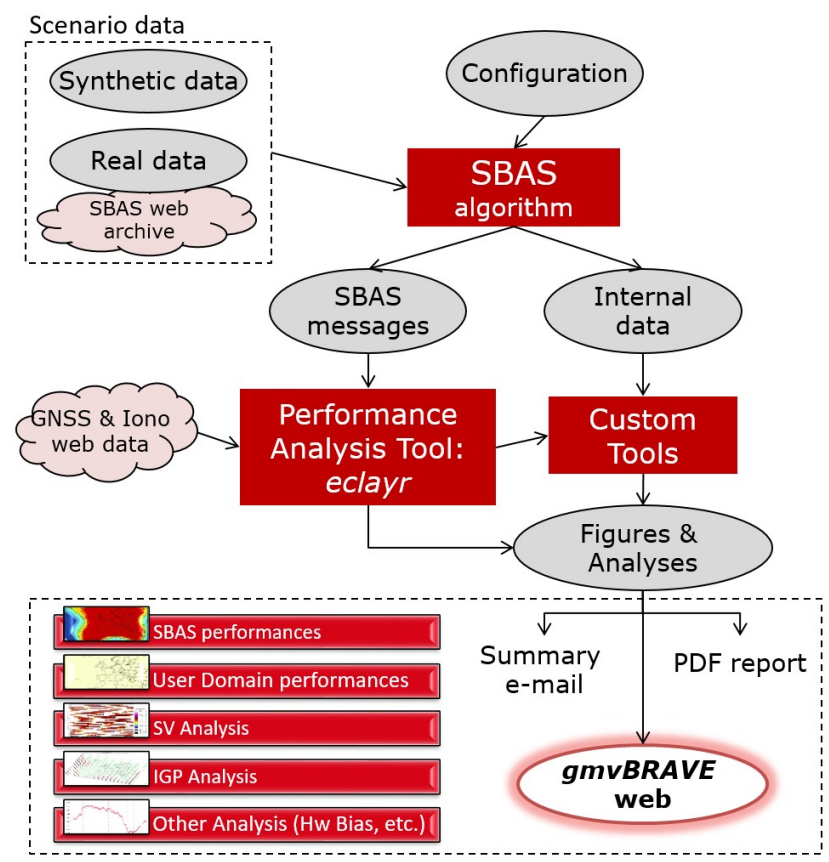

Figure 1 - Workflow of $g m v B R A V E$

\section{WEB INTERFACE \& DATA DISPLAY}

The amount of results from SBAS performances in form of plots and data tables can be very large and overwhelming to analyze. For this reason, $g m v B R A V E$ has been designed to incorporate a web interface where the extensive performance analysis is displayed in a very visual and structured way. gmvBRAVE's core process manages constantly the interaction between the analysis tools executions and its own online platform, in order to upload continuously all the results to the web interface.

In general, the web interface offers to its users:

- User-friendly design;

- Welcome page including a list of all available scenarios and a date-picker calendar to preview all archived daily analyses (Figure 2);

- Summary of analyzed performance for each day of a scenario, with the possibility for customizing displayed information with availability maps, satellite and Ionospheric Grid Point (IGP) monitoring figures, integrity ratios, diagrams and more;

- Launch information including selected tools used in the execution;

- Comparison between SBAS algorithm versions at a glance;

- Comparison with real system performances;

- Detection of anomalous events in a few mouse clicks;

International Technical Symposium on Navigation and Timing (ITSNT) 2018

13-16 Nov 2018

ENAC, Toulouse, France 
- Complete analysis report, per day and SBAS version, that can be downloaded in PDF format;

- Possibility of attaching own internal analysis reports performed by the development and maintenance teams;

- Computation of global scenario statistics over long time periods, shown in form of interactive graphs and data tables;

- Live information of SBAS execution (Figure 3) and eclayr queues status (Figure 4). The website also allows the possibility of visualizing a list of the algorithms and analysis tools being executed at the current time. For the eclayr tool also is available a screen to monitor the execution queues in the different servers;

- Additional scenario information: algorithmic versions used over time, featured days (e.g. days where a satellite mask change or the attachment of an internal analysis report were done) emphasized with colours (orange and blue, respectively; see Figure 2) and more.

Furthermore, platform users can be notified through a summary e-mail once the analysis has finished and the information has been displayed in the website. Development and maintenance teams can configure the content of this email, whose purpose is to collect, for all versions executed, a summary of the daily analysis performance, being this completely customizable according to the user's needs.
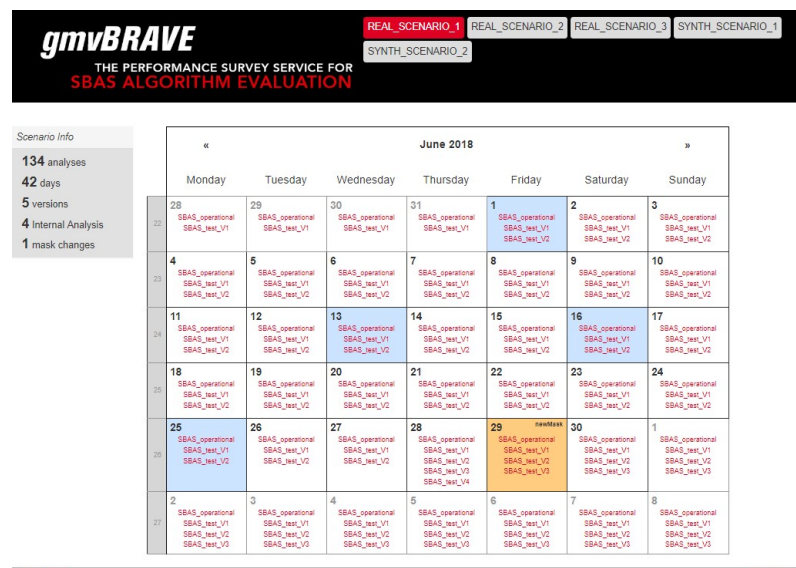

Figure 2-gmvBRAVE's navigable calendar

\subsection{Daily analysis}

gmvBRAVE delivers a complete analysis of relevant SBAS parameters for every day of a given scenario (both real or synthetic). In the main page of each scenario, the web interface shows a preview calendar with the available days in which the analysis has been performed (see Figure 2). Once a day is selected, a brief summary of the analysis shows up, offering the platform users a quick comparison between different SBAS versions by displaying them in column-formatted fashion where each column shows the results of a given version (Figure 5).
A complete analysis per version is performed, including:

- SBAS Signal In Space (SIS) analyses:

○ IGP analysis:

- IGP monitoring

- Grid of Ionospheric Vertical Delay (GIVD) error vs. Grid Ionosphere Vertical Error (GIVE) analysis

- GIVD error evolution

○ Space Vehicle (SV) analysis:

- SV monitoring

- Satellite Residual Error for the Worst user location (SREW) vs. User Differential Range Error (UDRE) analysis

- SREW evolution

- UDRE index evolution

○ Additional information from SBAS messages.

- SBAS performances extrapolated to the User Domain, illustrated through availability, continuity, accuracy and integrity maps.

- Analysis of internal algorithm information:

- Number of input measurements

- Hardware (HW) biases

- Clock errors, offsets and drifts

- Orbit determination errors

- Ionosphere analysis

- Vertical and horizontal errors per monitoring receiver

- Quality of service

$\circ$ And many more

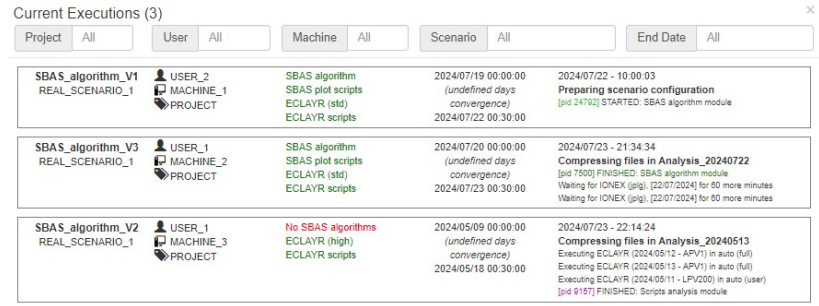

Figure 3 - List of current executions

ECLAYR machines queue (8)

Last updated on Friday Apr 19 14:00:37

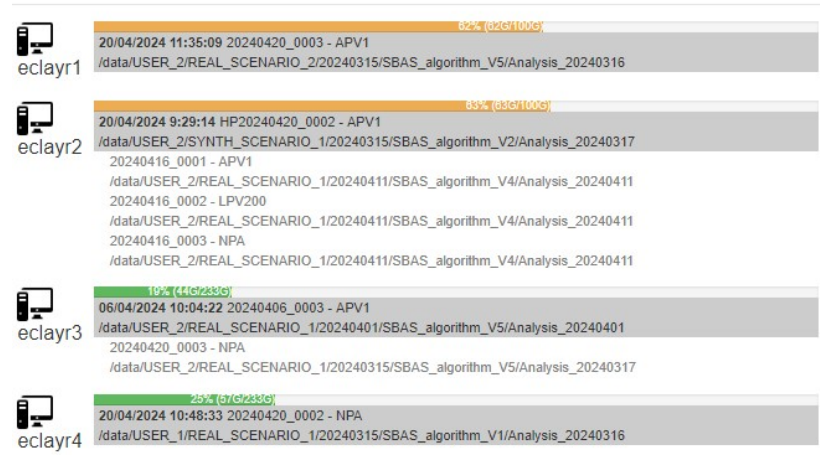

Figure 4 - eclayr queues in different servers

International Technical Symposium on Navigation and Timing (ITSNT) 2018

13-16 Nov 2018

ENAC, Toulouse, France 


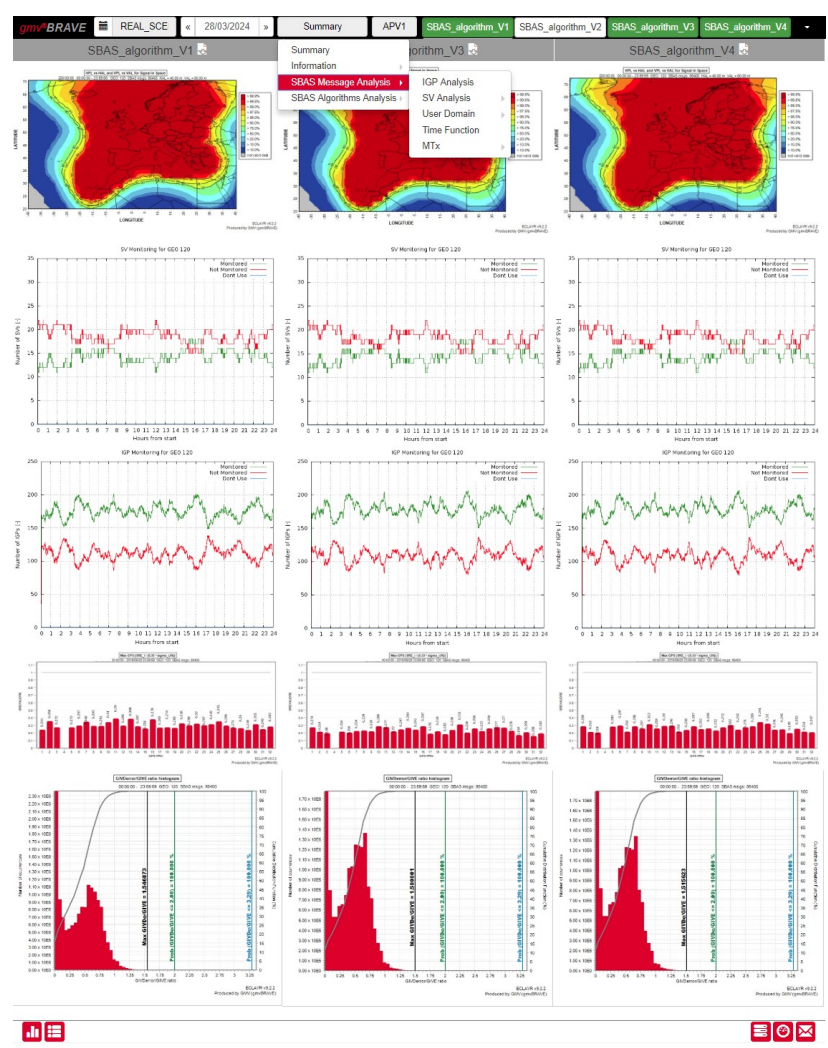

Figure 5-gmvBRAVE's daily summary page for SBAS algorithm comparison

To ease the visualization of all this information, the daily analysis page incorporates easy navigation tabs on top and shifting bars for:

- Choosing between different analysis categories (at pseudorange, user or algorithmic level) and service levels (NPA, APV-I, LPV-200, etc.);

- Hiding/showing to easy the visualization at the time of comparing the figures from the different algorithm versions of a SBAS;

- Scrolling through hourly figures to see the evolution of the data during the day (only available for specific sets of figures);

- Filtering data by independent satellites and the possibility of moving between different constellations;

- Filtering data by receivers;

- Moving forward and backward by day, or the possibility to choose a concrete day of the scenario through a drop-down calendar;

- Going back to preview calendar;

- Navigating quickly between different scenarios.

\subsection{Historical of global statistics}

As information from the daily analyses becomes available, gmvBRAVE tracks certain performance parameters relevant in a SBAS, elaborates daily data tables with statistics of these parameters and displays their temporal evolution using dynamic graphs (in the sense that both axes can be stretched or enlarged, e.g. to focus on smaller or larger time periods or different intervals of the parameter to study). This allows a broader overview of the system performances over periods of months or even years and allows the identification of seasonal or repetitive behaviours. The graph also includes tabs for displaying different statistics and for different services (e.g. APV-I).

An example of a historical tracking is shown in Figure 6 where the number of monitored IGPs with a GIVD error root mean square (RMS) $<0.5 \mathrm{~m}$ is shown for a period of about two years. As it can be seen, these historical graphs allow displaying statistics from different SBAS versions, identified with lines of different colours, that can be also dynamically hidden and shown as desired by the user.

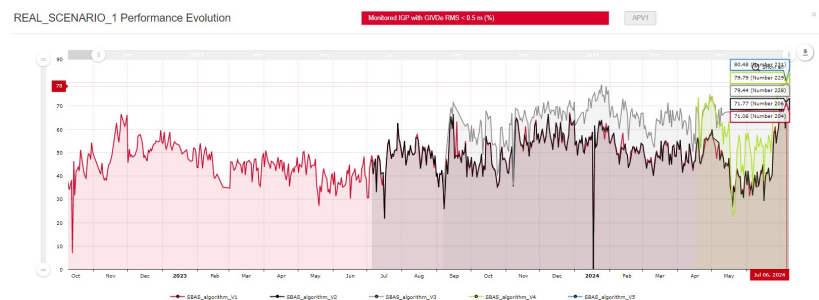

Figure 6-gmvBRAVE's historical performance graph

\section{EXAMPLES OF USE}

Practical examples of the usage of gmvBRAVE are given in this section. As it will be shown, the most common one falls back onto the comparison of versions. To do that, the website platform allows the possibility of enlarging and swapping figures in order to study possible differences between the performances of many SBAS algorithm versions. From this, gmvBRAVE has some essential uses that are described in the following.

\subsection{SBAS engineering activities}

gmvBRAVE has been designed mainly to simplify the maintenance of any SBAS system and the analysis of its performances by reducing the time associated to repetitive tasks like the organization and execution of several tools.

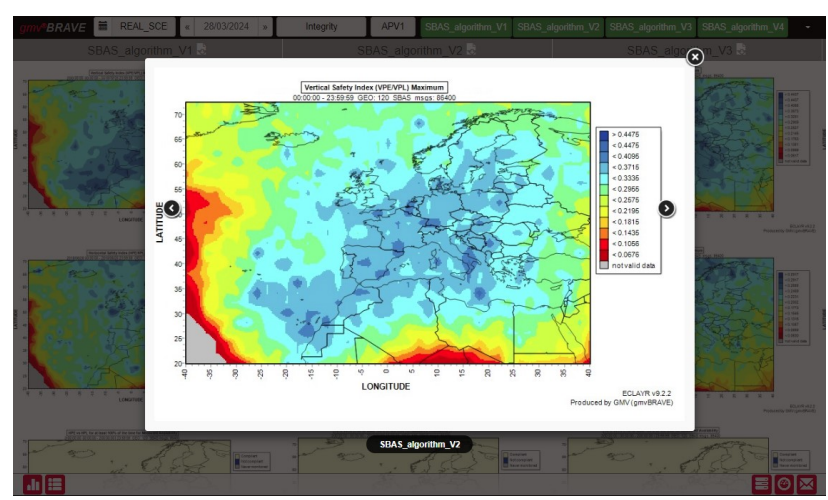

Figure 7 - Expanded plot (integrity map) with arrows for going from one SBAS version to another

Basically, the comparison of different SBAS algorithms at a glance (see e.g. Figure 5) that can be executed with both the same configuration and using same data, allows a quick analysis of the algorithms behaviour in a particular scenario. Thanks to the built-in tabs the user can hide and

International Technical Symposium on Navigation and Timing (ITSNT) 2018

13-16 Nov 2018

ENAC, Toulouse, France 
show different SBAS versions (in Figure 5, see green and white tabs in the upper bar of the website interface to indicate those shown and hidden versions, respectively) and make a proper comparison between the desired versions. By clicking on it, the user can expand any figure to improve its resolution and ease its visualization (Figure 7). Besides, the platform user can compare the differences between shown versions by using the arrows that appear on both sides of the figure.

\subsection{Impact analysis of anomalous events}

The preliminary analysis and identification of abnormal events that affect the behaviour of the algorithms and degrade the performances of the SBAS are of extreme interest for the operation and maintenance of the system, but also for the identification of candidate scenarios with which to develop and test future algorithm evolutions. Thanks to the amount of information displayed by the website interface and its easy navigability, gmvBRAVE allows the immediate detection of these types of events and their potential causes.

The list of these anomalous events is very large and its appearance can be a symptom of the non-robustness of algorithms against known physical phenomena or possible system failures. They include, for instance, ionospheric effects (e.g. scintillation or large gradients), degradation of the receiver environment, algorithm limitations, etc. As it will be shown in the following, by giving a couple of examples, the identification and analysis of any of these events can be easily done using gmvBRAVE.

\subsubsection{Loss of ionosphere monitoring}

Left column in Figure 8 shows an example of the detection of an event of loss of ionosphere monitoring caused in this particular case by some specific conditions of the ionosphere.

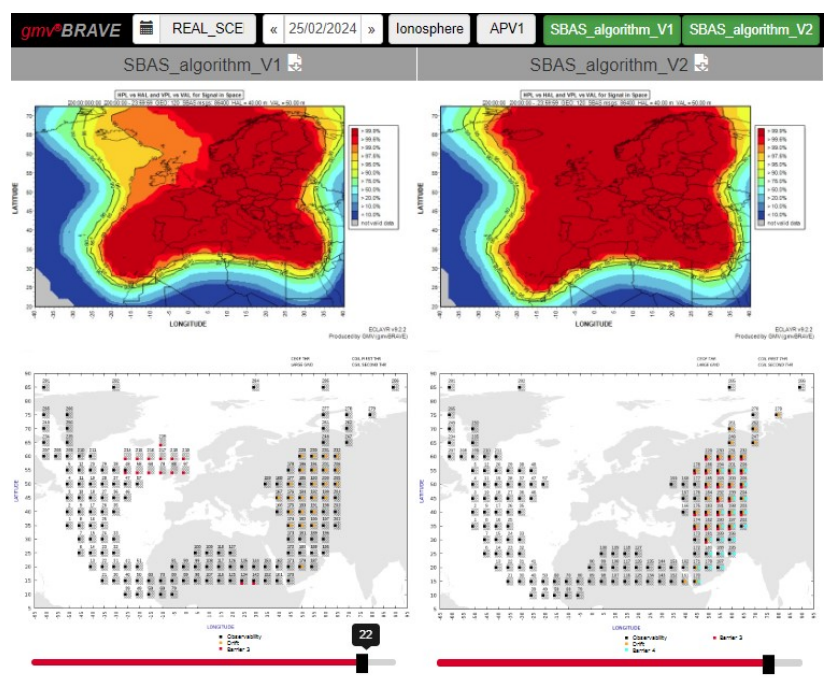

Figure 8 - Detection of a loss of ionosphere monitoring (left column) and comparison with an improved algorithms version that solve the issue (right column)

On the upper left-hand side plot of Figure 8, the availability map for APV-I service shows that there is a degradation of the performances (shown on the left side of the figure with yellow and orange colours; red colour indicates full system availability) with SBAS_algorithm_V1. By moving to that section in the website where the ionospheric analysis is displayed, the platform user can figure out the reason of such degradation: there are some IGPs that have been set to Not Monitored between hours 22 and 23 of the day by a given algorithmic barrier (see lower part of the Figure 8; the small black bar has been scrolled to show the IGP map between 22 and 23 hours). The result is that these IGPs have not been monitored (and then not available for SBAS users in that region in order to obtain ionospheric corrections) during a certain time period, degrading the overall availability (reflected in the map) of the SBAS service during the day.

\subsubsection{HW bias estimation event}

Left column in Figure 9 shows an example of the detection of an event caused by an issue in the estimation of inter-frequency HW biases that affects several satellites and receivers.

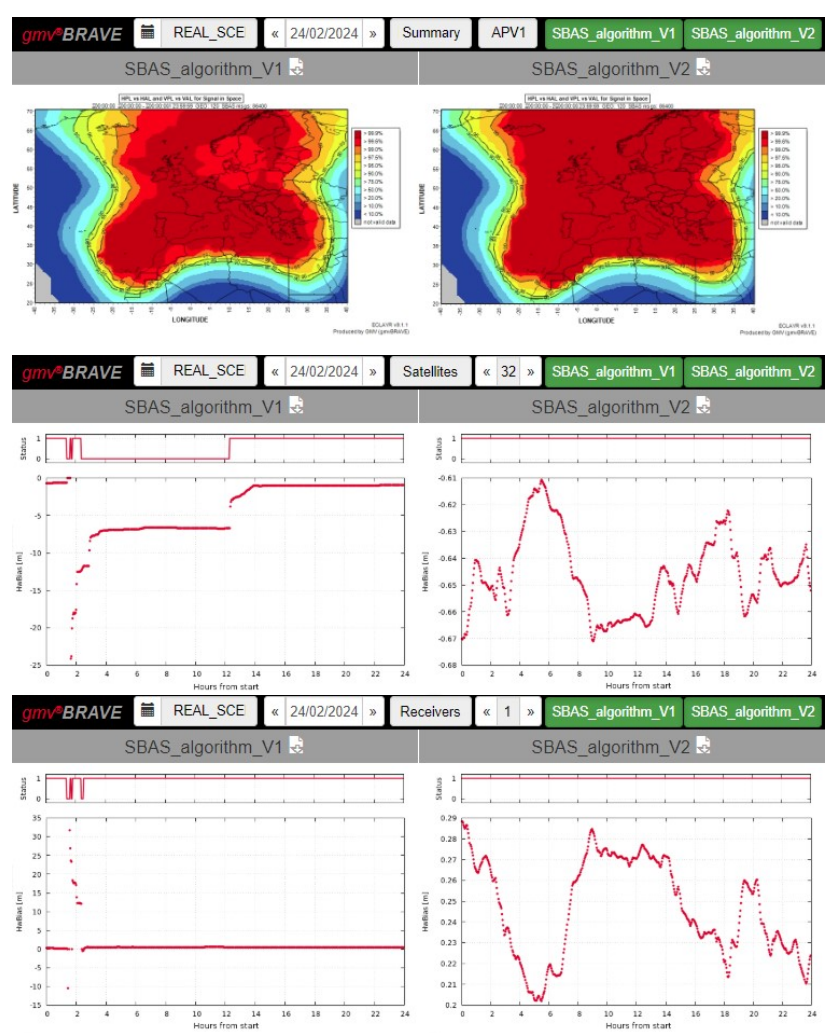

Figure 9 - Detection of a HW bias estimation event (left column) and comparison with an improved algorithms version that solve the issue (right column)

On the upper left-hand side plot of Figure 9, the availability map (APV-I service) shows that there is a degradation of the performances (shown on the center of the figure with light red colour) of SBAS_algorithm_V1. By moving to the section where the HW $\bar{b}$ ias analysis of all satellites is displayed, and filtering by satellite, the platform user can figure out that some issue is affecting to the convergence of the HW biases of a few satellites (center plot in Figure 9) after their reset, since HW biases are taking very large values (in absolute value; see the negative peak in the figure), becoming invalid and

International Technical Symposium on Navigation and Timing (ITSNT) 2018

13-16 Nov 2018

ENAC, Toulouse, France 
holding this state for many hours through the day after they finally converge to the similar values they had before the reset. Filtering by receiver, the user can observe that the same issue is happening in a few receivers (lower plot in Figure 9). This quick analysis allows to the user to figure out that a given barrier in the algorithms is being activated for the lines of sight given by these satellites and receivers, eventually inducing the reset of $\mathrm{HW}$ bias estimation.

\subsection{Testing of SBAS algorithm improvements}

The website interface of $g m v B R A V E$ is the perfect platform for tool users, and more widely to development and maintenance teams, to make quick comparisons between different SBAS algorithms executions. It has been already mentioned that users can compare different executions of a same SBAS algorithm in which different configurations were used or, even more relevant, the platform offers the possibility of comparing between different SBAS algorithm versions. This latter comparison has been revealed to be one of the gmvBRAVE's strengths since it makes possible to develop SBAS algorithm improvements in parallel to the maintenance tasks that are required by the operational version. This allows looking for those algorithms that are robust against anomalous events with the aim of improving the global performances of the SBAS. The platform facilitates that these prototype versions can be tested continuously for very long periods (up to years) before their final implementation, being able to use for that purpose the same real data that the operational version uses, so that a direct comparison between versions can be made. Following the examples already introduced in the section 5.2, the process of testing experimental versions of SBAS algorithms is illustrated here.

In the left column of Figure 8, an ionospheric anomalous event that was degrading the performances of the SBAS algorithm version, referred as SBAS_algorithm_V1, was shown. In the right column an experimental version, referred as SBAS_algorithm_V2, which includes some improvements to make the algorithms robust against this particular event. As it can be seen in the availability map (upper right-hand side plot) the performance is highly improved, respect to that of SBAS_algorithm_V1, on the left side of the map (now it is in red colour) since now no IGP has been set to Not Monitored over such area (see lower right-hand side plot).

In the left column of Figure 9 a HW bias estimation event that was also causing a degradation of the performances of algorithm SBAS_algorithm_V1 was shown. In the right column the experimental version SBAS_algorithm_V2 also includes an improvement on algorithms to avoid this particular issue. The availability map (upper right-hand side plot) reveals that the performance has been improved in the central region of the map. Likewise, the HW bias plots for both the satellite (center right-hand side plot) and the receiver shown (lower right-hand side plot) reveal that now the algorithm is robust against this issue since no reset is detected and the estimated biases show small variations during the whole day.

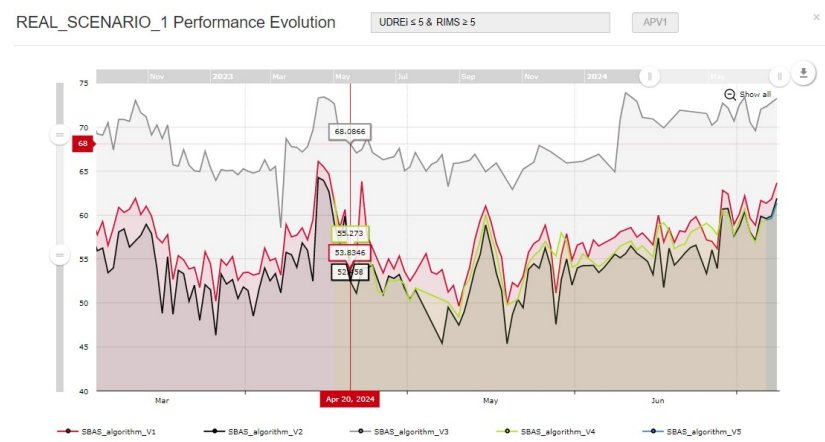

Figure 10 - Historical performance: evolution of the percentage of satellites with UDREi $\leq 5$ and in view of at least five receivers

An additional example is shown in the following to illustrate that the global statistics graphs also help to track other algorithm issues that may be improved by experimental versions. Figure 10 shows the percentage of satellites with UDREi $\leq 5$ and in view of at least five receivers, as a function of time. In this case, version SBAS_algorithm_V3 is identified to be that one which improves the most the compliance to the UDRE performance requirement since it achieves larger values for the time interval displayed in the figure.

\subsection{Catalogue of anomalous events}

An additional service provided as part of $g m v B R A V E$ is the generation of a database of real system events observed in the real SIS together with the corresponding system behaviour. This information constitutes a catalogue of days affected by anomalous events (e.g. ionospheric perturbations, satellite anomalies, etc.) in order to characterize the cause/effect relationship in each of them. This information can be then use for future system qualification campaigns.

\section{CONCLUSIONS}

SBAS development and maintenance activities require the analysis of lots of performances results. Developed by GMV, the $g m B R A V E$ platform for SBAS engineering has been presented as the tool capable of dealing with all the amount of results that any SBAS can provide. This fact allows gmvBRAVE to be used both as a monitoring and analysis tool for daily SBAS performances, as well as for testing algorithmic experimental evolutions of a SBAS.

As it has been shown in the paper, $g m v B R A V E$ aids the execution of SBAS algorithms and performance executions and tests, providing relevant results in the form of plots and tables and easing its analysis through a friendly web interface. The generation of extensive analysis reports allows platform users to make immediate, preliminary analysis for tracing the causes and origins of anomalous events or unexpected algorithm behaviours, in order to find algorithmic modifications that will improve the system performances in these situations. For such 
purpose, a web interface has been designed to ease the comparison of results between different evolutions of a same SBAS algorithm. Indeed, the simultaneous production of results for different algorithm executions (using same real data for long periods of time) and the possibility of its quick comparison is one of the cornerstone points of $g m v B R A V E$, turning it into an excellent platform not only for maintenance stages but for development campaigns where validation times seek to be considerably reduced.

The tool has been satisfactorily tested in different practical examples of use, showing in first place the main advantages of using it as a tool for maintenance tasks of an operational SBAS. Furthermore, it has been shown that, with a tiny effort, anomalous events can be analyzed and identified. Finally, it has been shown to be extremely valuable as a tool for testing algorithmic experimental versions prior to a final system deployment.

In the new era that is coming for SBAS, the existence of a tool that allows continuous monitoring and analysis of such systems is a must. Throughout this paper, it has been proved that $g m v B R A V E$ is the tool that perfectly fits with the fulfillment of all this tasks for SBAS engineering, either during its development, deployment or operational stages.

\section{REFERENCES}

[1] GMV, gmvBRAVE [Online], https:/www.gmv.com/en/Products/brave/

[2] GMV, EETES [Online], https:/www.gmv.com/en/Sectors/space/Satellite_navigati on_systems/GNSS_tools_development.html

[3] GMV, magic $S B A S$ [Online], https://www.gmv.com/en/Products/magicSBAS/

[4] GMV, eclayr [Online], https:/www.gmv.com/en/Products/eclayr/

[5] EGNOS Data Access Service (EDAS) [Online], https://www.gsa.europa.eu/egnos/edas 